\title{
Water-Splitting Photoelectrolysis Reaction Rate via Microscopic Imaging of Evolved Oxygen Bubbles
}

\author{
Andrew J. Leenheer ${ }^{\mathrm{z}}$ and Harry A. Atwater
}

Thomas J. Watson Laboratories of Applied Physics, California Institute of Technology, Pasadena, California 91125, USA

Bubble formation and growth on a water-splitting semiconductor photoelectrode under illumination with above-bandgap radiation provide a direct measurement of the gas-evolving reaction rate. Optical microscopy was used to record the bubble growth on single-crystal strontium titanate immersed in basic aqueous electrolyte and illuminated with UV light at $351 / 364 \mathrm{~nm}$ from a focused argon laser. By analyzing the bubble size as a function of time, the water-splitting reaction rate was determined for varying light intensities and was compared to photocurrent measurements. Bubble nucleation was explored on an illuminated flat surface, as well as the subsequent light scattering and electrode shielding due to the bubble. This technique allows a quantitative examination of the actual gas evolution rate during photoelectrochemical water splitting, independent of current measurements. (c) 2010 The Electrochemical Society. [DOI: 10.1149/1.3462997] All rights reserved.

Manuscript submitted April 13, 2010; revised manuscript received June 16, 2010. Published July 19, 2010.

The production of hydrogen using solar energy to directly split water in a semiconductor photoelectrochemical cell is a promising source of carbon-free fuel, ${ }^{1}$ but many issues with the semiconductor-liquid interface remain. Semiconductors with bandgaps $\sim 2 \mathrm{eV}$ useful for absorbing the solar flux and possessing the necessary potential to split water ${ }^{2}$ often suffer stability issues due to photoexcited charge carriers corroding the semiconductor rather than transferring into the electrolyte. ${ }^{3}$ Adding heterogeneous catalysts or structuring the electrode surface can provide more active sites for charge transfer, lowering the overpotentials required and possibly increasing stability, but often the nature of the active sites is unknown. ${ }^{4-9}$ Traditional experimental techniques involve modifying or structuring the entire surface of a semiconductor photoelectrode and measuring the photocurrent under illumination; this method provides only the average reaction rate of all the various redox reactions occurring over the entire exposed surface. Localized methods that differentiate the activity on separate patterned areas on the same electrode could offer a wealth of information about the surface sites important for efficient charge transfer. Previous research has focused on utilizing a scanned electrochemical probe near the surface ${ }^{10}$ that can even include local illumination through an optical fiber, ${ }^{11}$ a very useful but rather perturbative and difficult method to implement.

Examining the evolved gas bubbles could provide this type of local measurement by recording their growth rate at different surface features. Additionally, measuring the bubble and hence the actual gas produced differentiates between the gas-evolving reaction of interest and any parasitic corrosion reactions that can still contribute to the measured current. Optical microscopy of the photogenerated gas bubbles during their growth is a straightforward technique and provides a localized measurement of the reaction rate at various areas on a semiconductor surface.

As a model system to explore the use of this technique, we used n-type $\mathrm{SrTiO}_{3}$ as a semiconductor photoanode to split water while observing the resulting oxygen bubbles with an inverted optical microscope. $\mathrm{SrTiO}_{3}$, while not suitable for solar applications due to its wide $3.2 \mathrm{eV}$ bandgap, ${ }^{12}$ is stable in aqueous environment with the conduction band energy high enough to reduce water to hydrogen gas. ${ }^{13,14}$ The lack of corrosion means that a simultaneous measurement of the current and bubble growth allows a comparison of the two methods. Because visible light does not generate charge carriers, the microscope illumination used for imaging is decoupled from the above-bandgap UV laser illumination, allowing the bubble nucleation and growth dynamics to be easily observed. We found that, indeed, the water-splitting reaction rate can be locally and quantitatively determined from the recorded bubble size as a function of time.

${ }^{\mathrm{z}}$ E-mail: ajl@ caltech.edu

\section{Experimental}

Single crystals of $\mathrm{SrTiO}_{3}(100)$ were obtained from MTI Corp. and were colorless and transparent upon arrival. Samples were annealed at $1000^{\circ} \mathrm{C}$ under $5 \% \mathrm{H}_{2} / 95 \% \mathrm{~N}_{2}$ until they were bluish black but still somewhat transparent, resulting in n-type conductivity. ${ }^{15}$ A schematic of the experimental setup is shown in Fig. 1, and a picture of the actual cell is included in the supplemental material (Fig. S1). ${ }^{16}$ Electrochemical cells were fabricated by contacting the edges of a sample with indium gallium eutectic, silver adhesive paste, and a copper wire, then sealing the sample to the bottom of a plastic Petri dish with insulating epoxy (Torrseal, Varian Inc.), leaving only $\sim 10 \mathrm{~mm}^{2}$ of the $\mathrm{SrTiO}_{3}$ surface exposed to the electrolyte. After the epoxy cured, the sample was rinsed clean with isopropyl alcohol and deionized $\mathrm{H}_{2} \mathrm{O}$, and blown dry with a $\mathrm{N}_{2}$ stream. About $10 \mathrm{~mL}$ unstirred, oxygen-saturated reagent grade 1.6 $\mathrm{M} \mathrm{KOH}$ in deionized water was used as the electrolyte, and the cell was set up in the three-electrode configuration with a Pt gauze counter electrode and $\mathrm{Ag} / \mathrm{AgCl}$ in $3 \mathrm{M} \mathrm{NaCl}$ reference electrode. A positive bias of $200 \mathrm{mV}$ vs $\mathrm{V}_{\mathrm{Ag} / \mathrm{AgCl}}$ was applied to the sample with a Gamry Reference 600 potentiostat to ensure solely $\mathrm{O}_{2}$ evolution and maximize quantum yield at the $\mathrm{SrTiO}_{3}$ surface, and the current flow was recorded using the potentiostat. An oxygen atmosphere was maintained by flowing pure oxygen into a transparent plastic enclosure that housed the entire cell. Photocurrent was measured by chopping the illumination at $5 \mathrm{~Hz}$ and measuring the current using an SRS SR830 lock-in amplifier.

The bubble evolution was observed in a Zeiss Axio Observer inverted microscope equipped with a $10 \times$ objective. Samples were illuminated from above with both transmitted visible light via a halogen lamp filtered through a $430 \mathrm{~nm}$ long-pass filter and abovebandgap radiation via the $351 / 364 \mathrm{~nm}$ lines of an $\mathrm{Ar}^{+}$laser focused to a small spot on the sample surface. The laser power was attenuated using neutral density filters and measured at the sample position with a Newport 1835-C power meter equipped with a UV-calibrated Si photodiode, and the power was monitored throughout the experiment. Videos were recorded at 30 frames/s using a black-and-white digital camera attached to the microscope. The laser spot size at the sample surface was measured using the camera, fitting the intensity to a Gaussian profile, and using the $1 / \mathrm{e}$ value as the spot radius, $r_{\text {laser }}=20 \mu \mathrm{m}$. The laser beam was manually shuttered to control bubble evolution, and the microscope focus was slightly adjusted during growth to keep the outer bubble edges in focus.

Calculations were performed using either IGOR Pro or Mathematica software on a personal computer.

\section{Results}

Illuminating the biased $\mathrm{SrTiO}_{3}$ surface with UV laser light resulted in a few microamps of photocurrent, while the contribution from the visible halogen lamp illumination to the photocurrent was 


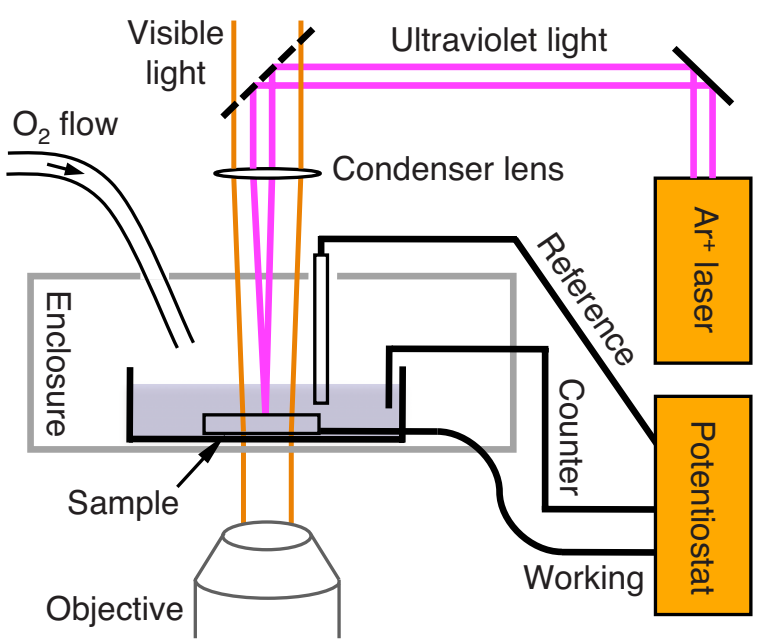

Figure 1. (Color online) Schematic of the experimental setup. The sample was sealed to the bottom of a Petri dish filled with electrolyte, and the entire cell was enclosed in a transparent plastic box to maintain an oxygen atmosphere. A cube beam splitter combined the visible illumination and UV laser.

negligible. Focusing the laser to a $20 \mu \mathrm{m}$ radius spot with at least $37 \mu \mathrm{W}$ beam power resulted in a single bubble nucleating and growing at the laser spot; however, lower intensities produced photocurrent but no bubble, while extremely high intensities resulted in copious, uncontrolled bubbling and noticeable local sample degradation. For conditions of single bubble growth, a representative time series of the photocurrent, bubble radius, and calculated gas present in the bubble is shown in Fig. 2. The video of bubble growth was digitally recorded and analyzed using image thresholding within IGOR Pro software to extract the bubble radius $r_{\text {bub }}$ from each frame, and the processed video corresponding to Fig. 2 is included in the supplemental material (movie S1) ${ }^{16}$ as well as the frame processing workflow (Fig. S2). ${ }^{16}$ An example of the bubble image and software edge recognition is shown in the inset of Fig. 2; the bright spot seen in the bubble center is a result of the optical scattering of the bubble. Assuming for each bubble $(i)$ an approximately spherical shape, (ii) a composition of only gaseous oxygen and water mol-

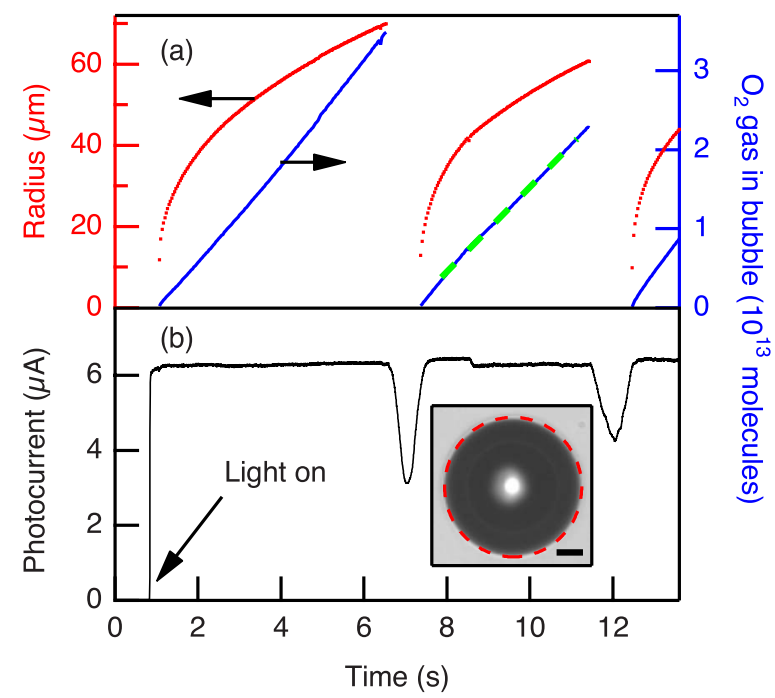

Figure 2. (Color online) (a) Measured bubble radius and calculated gas present, and (b) photocurrent as a function of time at an irradiance of 1.8 $\times 10^{5} \mathrm{~W} / \mathrm{m}^{2}$. The dotted line in (a) is the fit, whose slope gives the reaction rate. Inset: Example of an analyzed video frame with the bubble perimeter shown as a dotted line (10 $\mu \mathrm{m}$ scale bar).

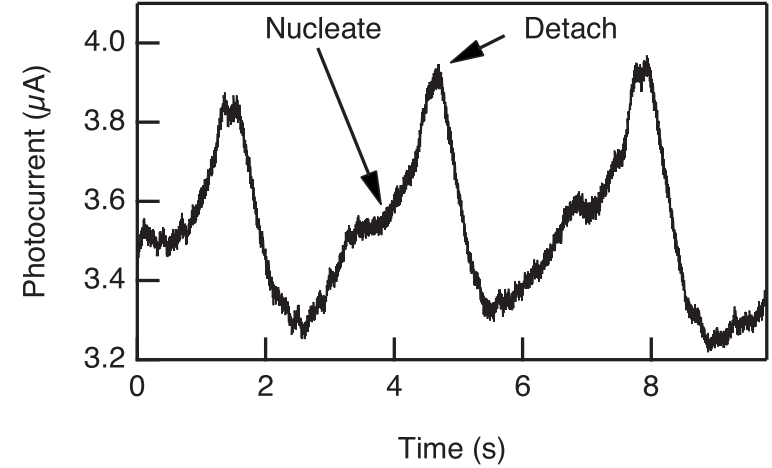

Figure 3. Photocurrent detail for a series of bubble nucleation, growth, and departure sequences at $1.1 \times 10^{5} \mathrm{~W} / \mathrm{m}^{2}$.

ecules, (iii) a temperature of $298 \mathrm{~K}$, and (iv) an internal pressure of $1 \mathrm{~atm}$ plus the Laplace pressure, the number of oxygen molecules inside the bubble was calculated using the ideal gas law

$$
N_{\mathrm{O}_{2}}=\frac{\left(P_{0}-P_{\mathrm{H}_{2} \mathrm{O}}+2 \gamma / r_{\text {bub }}\right)}{k T} \frac{4}{3} \pi r_{\text {bub }}^{3}
$$

where $P_{0}=1 \mathrm{~atm}$ is the ambient pressure, $P_{\mathrm{H}_{2} \mathrm{O}}=0.033 \mathrm{~atm}$ is the vapor pressure of water inside the bubble, ${ }^{17} \gamma=0.71$ atm $\mu \mathrm{m}$ is the gas/liquid surface tension, ${ }^{17} k$ is Boltzmann's constant, and $T$ $=298 \mathrm{~K}$ is the ambient temperature. At the counter electrode, bubbles were also evident that were presumably $\mathrm{H}_{2}$, but no attempt was made to identify the actual gas composition of any bubbles.

The rate of oxygen production at the photoanode and thus the water splitting rate could then be calculated in two ways: from either the photocurrent or the rate of bubble growth. For the traditional photocurrent measurement, Faraday's law gives the oxygen evolution rate

$$
R_{\mathrm{O}_{2}, \text { cur }}=\frac{I_{\text {photo }}}{q Z}
$$

where $I_{\text {photo }}$ is the photocurrent, $q$ is the charge of an electron, and $Z=4$ is the number of electrons transferred to evolve one oxygen molecule. Alternatively, the rate $R_{\mathrm{O}_{2}}$,bub can be calculated from the bubble growth by fitting a line to the $N_{\mathrm{O}_{2}}$ vs time plot, as shown by the dotted line in Fig. 2. The photocurrent varied upon bubble nucleation and detachment, as shown in Fig. 3. However, these variations were often small when compared to the overall photocurrent.

Within the intensity range that allowed single bubble nucleation and growth, the laser power was varied and the oxygen evolution rate averaged at various locations and for multiple bubbles on a single electrode; the results are shown in Fig. 4a. Here, the laser power was converted to an approximate irradiance by dividing by the spot area $\pi r_{\text {laser }}^{2}$ The minimum irradiance to nucleate a bubble was $\sim 3 \times 10^{4} \mathrm{~W} / \mathrm{m}^{2}$, where a few seconds of waiting time between bubbles existed. At irradiances below this, a bubble was first nucleated with more intense illumination, the laser was shuttered, the intensity was reduced, and the sample was again illuminated to measure the actual bubble growth. The remarkable agreement between the traditional current measurement and present bubble growth methods shows the utility of recording bubble growth as a metric of the actual gas evolved, which can be especially useful for semiconductors that are susceptible to photocorrosion. As a more direct comparison, the external quantum yield $\Phi$ shown in Fig. $4 b$ was calculated using

$$
\Phi=\frac{\text { no. of electrons utilized }}{\text { no. of incident photons }}=\frac{Z R_{\mathrm{O}_{2}}}{P_{\text {laser }} /(h c / \lambda)}
$$

where $\lambda \approx 360 \mathrm{~nm}$ is the laser wavelength, $P_{\text {laser }}$ is the laser power, $h$ is Planck's constant, and $c$ is the speed of light. The error bars 


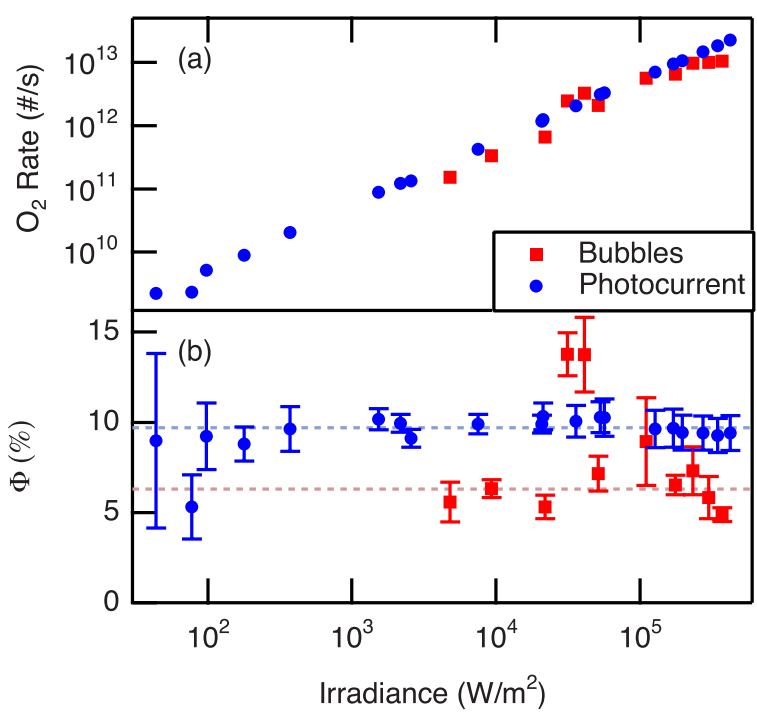

Figure 4. (Color online) (a) Oxygen production rate and (b) quantum yield $\Phi$ as a function of laser irradiance measured by the current (squares) and by the bubble growth rate (circles). The laser spot radius was $20 \mu \mathrm{m}$. The dotted lines indicate the approximate quantum yields measured by the two methods.

shown are the standard deviation of measurements done at multiple locations on the sample and with multiple bubbles. The error was higher for lower irradiances because of the difficulty in distinguishing the small photocurrent and low bubble growth rate from noise in the measurement.

\section{Discussion}

To quantify and analyze the gas evolution rate by examining bubble growth, conditions of single bubbles growing at the area of interest were desired. Bubble nucleation is highly dependent on the local properties of the surface, with pits and hydrophobic surfaces encouraging nucleation. ${ }^{18,19}$ However, we desired to decouple the sites of preferred bubble formation from the sites of enhanced gas evolution. Without introducing artificial nucleation sites nearby, it was necessary to use strong local irradiation to overcome the diffusion of dissolved gases into the bulk electrolyte and achieve the local supersaturation necessary to form a bubble. Thus, the irradiances seen in Fig. 4 are much higher than in typical semiconductor photoelectrolysis studies ${ }^{14,20}$ and certainly higher than the UV above-bandgap solar irradiance. Even so, the remarkable agreement between the measurement of photocurrent and bubble growth for quantifying the reaction rate shows the usefulness of this alternative method. The overall rate and quantum efficiency appears slightly lower for the bubble measurement due to the tendency of the gas to enter solution, either directly or via bubble shrinkage due to the Laplace pressure. Additionally, the photocurrent may be overestimating the gas evolution rate due to possible parasitic side reactions.

The small illuminated area and nonoptimized sample quality likely caused the overall low observed quantum efficiency $\Phi$. Some photogenerated carriers are always lost due to recombination across the depletion region facilitated by traps. When the dark junction area is much larger than the illuminated area, excess carriers can diffuse away from the laser spot and be lost across the dark junction, resulting in a considerable loss of photocurrent. At higher irradiances, other reasons for the low observed $\Phi$ likely include diffusionlimited current in the unstirred electrolyte, the relatively weak $\mathrm{OH}^{-}$ concentration $^{20}$ and low rates of charge transfer to oxidize $\mathrm{H}_{2} \mathrm{O}$, and possible Auger recombination under the high level injection conditions with strong illumination (see below). The observed $\Phi$ agreed fairly well at high irradiances with previously reported data in a similar experiment that used a much larger laser spot of $1 \mathrm{~mm} .^{14}$

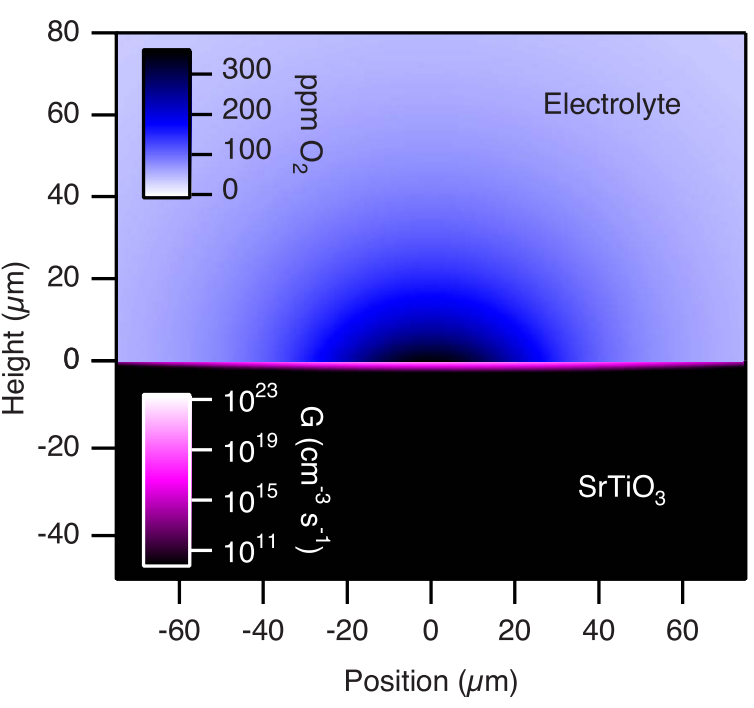

Figure 5. (Color online) Simulated steady-state radial profiles of the dissolved oxygen in electrolyte (top) and the carrier generation rate in $\mathrm{SrTiO}_{3}$ (bottom) for a Gaussian illumination profile. The laser irradiance was 3 $\times 10^{4} \mathrm{~W} / \mathrm{m}^{2}$ at a radius of $20 \mu \mathrm{m}$ with an assumed $\Phi=10 \%$.

To help analyze the conditions for bubble nucleation, simulations of the steady-state carrier generation rate in the semiconductor and dissolved oxygen profile in the electrolyte are shown in Fig. 5 for experimental conditions under which the laser irradiance was just at the threshold for bubble formation. In the semiconductor, the charge carrier generation rate was calculated from

$$
G_{\text {carrier }}=\frac{P_{\text {laser }} \lambda}{h c \pi r_{\text {laser }}^{2}} \alpha e^{-\alpha z} e^{-r^{2} / r_{\text {laser }}^{2}}
$$

where $\alpha=2.5 \times 10^{4} \mathrm{~cm}^{-1}$ is the absorption coefficient of $\mathrm{SrTiO}_{3}$ at $360 \mathrm{~nm} .{ }^{12}$ The calculations of dissolved oxygen in the electrolyte follow the treatment of Lax for a Gaussian laser profile ${ }^{21}$ details are given in the Appendix. The generation rate was over $10^{23} \mathrm{~cm}^{-3} \mathrm{~s}^{-1}$, well into high level injection conditions. Based on the measured $\Phi$, we assumed that $10 \%$ of the photogenerated carriers are successful in oxidizing water and evolving dissolved oxygen that then diffuses away from the laser spot, resulting in the shown dissolved oxygen profile in the electrolyte. As the saturation condition for oxygen in water at $1 \mathrm{~atm}$ oxygen is $\sim 40 \mathrm{ppm}$, these calculations indicate that a supersaturation of $\sim 8$ times the saturation limit is approached before bubble nucleation. This observation is consistent with previous studies in which the supersaturation near gas-evolving electrode surfaces is often $\sim 5$ times and as high as 100 times the saturation limit due to the energetic barriers involved in bubble nucleation at smooth surfaces. ${ }^{22-24}$ Indeed, the high concentrations of dissolved oxygen near the electrode surface can impede the water oxidation reaction, lowering $\Phi$.

Once a bubble forms, it provides a sink for the gaseous products, quickly consuming much of the nearby dissolved oxygen, especially under our experimental conditions where the laser is of comparable size or smaller than the bubble size. For the majority of a bubble's dwell time on the electrode, its radius as seen in Fig. 2 followed the relationship $r_{\text {bub }} \propto t^{1 / 3}$, indicating a "direct injection" of the evolved gas into the bubble from the electrode rather than bubble growth due to diffusion from the bulk electrolyte or liquid inertia. ${ }^{25}$ However, near the limit of bubble nucleation, the relatively long waiting time between bubbles caused a buildup of dissolved gas and high bubble growth rate once nucleated, resulting in the higher apparent quantum efficiencies for laser irradiances near $\sim 4 \times 10^{5} \mathrm{~W} / \mathrm{m}^{2}$, as seen in Fig. 4b. For these "nucleation-limited" conditions, the bubbles grew faster than the oxygen production at the electrode surface. 
It is useful to examine the assumptions made and possible associated errors in the simple calculation we used to quantify the amount of oxygen evolved. First, the ideal gas law was invoked for the bubble, while a more accurate calculation could use the van der Waals equation

$$
\left(P+\frac{n^{2} a}{V^{2}}\right)(V-N b)=N k T
$$

where $a$ and $b$ are the van der Waals parameters for oxygen. Given the approximate pressure and volume of a typical bubble, the difference in the number of gas molecules for the ideal and van der Waals cases was on the order of $1 \%$, not large enough to be significant. Second, the partial pressure of oxygen in the bubble was corrected for the vapor pressure of water and the Laplace pressure (a small correction at a bubble radius greater than a micrometer or two), and the extra gravitational pressure of the liquid above the bubble was considered to be negligible. Though the gas composition was not measured, it is reasonable to assume that it is mostly oxygen because the standard model for photoelectrochemical cells ${ }^{26}$ predicts that only holes will be available at the photoanode surface (especially with the extra $+200 \mathrm{mV}$ vs $\mathrm{V}_{\mathrm{Ag} / \mathrm{AgCl}}$ applied bias) therefore only oxidizing water, not reducing it at $\mathrm{SrTiO}_{3}$. Third, the bubble volume was calculated assuming a sphere, though an actual bubble will have a nonzero contact angle measured through the liquid and more of an oblate spheroid shape. However, we have estimated that the contact angle was small, perhaps $\sim 5^{\circ}$ at most (see below), so the amount of volume cutoff by the solid interface was $\sim 10^{6}$ times smaller than the bubble volume, a very small correction. Also, previous studies that observed bubbles at water-splitting electrodes from the side show very close to a spherical shape. ${ }^{27}$ Fourth, the temperature in the bubble was assumed to be ambient temperature, but the laser could possibly cause local heating and vaporization. Because the water and gas do not absorb the UV laser, the primary rise in temperature would be in the semiconductor. The temperature profile in a semiconductor irradiated with a Gaussian laser profile has been solved by $\operatorname{Lax}^{21}$ with the maximum temperature rise $\Delta T_{\max }$ at the center of the laser spot given in the Appendix. Using $K$ $=11 \mathrm{~W} \mathrm{~m}{ }^{-1} \mathrm{~K}^{-1}$ as the thermal conductivity of $\mathrm{SrTiO}_{3},{ }^{28}$ the maximum temperature rise was only $0.64{ }^{\circ} \mathrm{C}$ even at the highest laser irradiances used $(500 \mu \mathrm{W}$ at $20 \mu \mathrm{m}$ beam radius), not large enough to introduce a significant error in the gas calculation. Also, this calculation assumed no heat transfer to the liquid so the temperature rise will be even lower. The experimental uncertainties arising from averaging multiple measurements, calibrating distances in the microscope, and keeping the edge of the bubble in focus were the primary sources of error.

The primary drawback to this technique was the interference of the bubble with the area of study. Once a bubble was formed, the existence of a finite contact angle means that an area of the electrolyte/solid interface was excluded; unfortunately, the same area was illuminated by the focused laser beam. The contact angle, though difficult to measure in situ with our experimental setup, can be estimated by balancing the buoyancy force of the bubble at its break-off radius in quiescent liquid with the adhering force resulting from surface tension. The equation obtained is the so-called Fritz equation for the contact angle $\theta$ in degrees ${ }^{29}$

$$
\theta \approx 46.8 r_{\text {depart }} \sqrt{\frac{g\left(\rho_{\mathrm{L}}-\rho_{\mathrm{G}}\right)}{\gamma}}
$$

where $r_{\text {depart }}$ is the bubble radius at departure, $g$ is the acceleration due to gravity, $\rho_{\mathrm{L}}$ and $\rho_{\mathrm{G}}$ are the densities of the liquid and gas, respectively, and $y=0.072 \mathrm{~N} / \mathrm{m}$ is the surface tension between the liquid and gas. ${ }^{17}$ For the typical observed break-off radii of $30-60 \mu \mathrm{m}$, the calculated contact angle was $0.5-1^{\circ}$, and the radius of the excluded photoelectrode area was thus $0.25-1 \mu \mathrm{m}$. Occasional larger bubbles of $200 \mu \mathrm{m}$ radius shielded up to a $20 \mu \mathrm{m}$ radius area. This measurement of excluded area is comparable to the gas/solid interfacial area evident in the microscope for a large

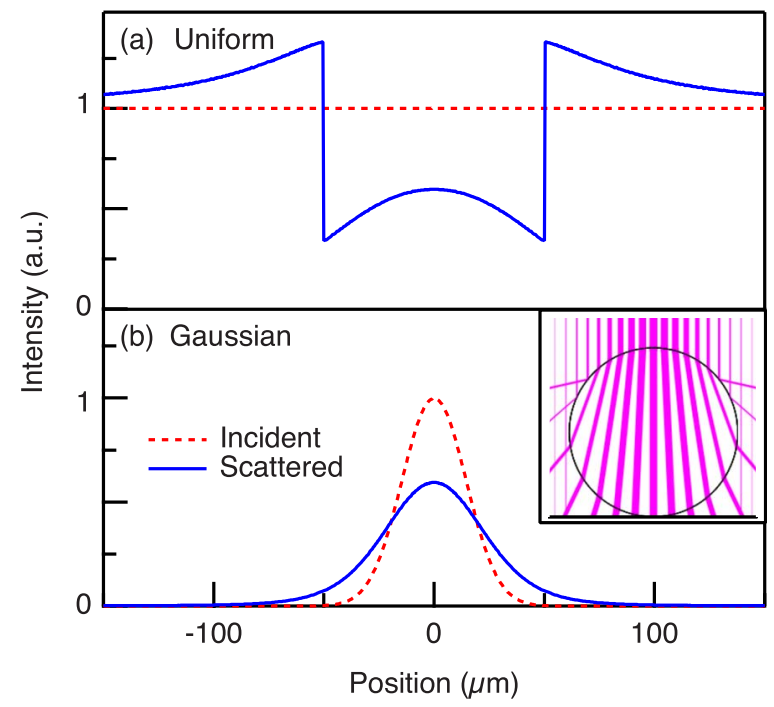

Figure 6. (Color online) Simulations of the radial laser profile after scattering by a $50 \mu \mathrm{m}$ radius bubble for (a) a uniform incident profile and (b) a Gaussian incident profile with a $20 \mu \mathrm{m}$ radius. Inset: Schematic of rays passing through the bubble.

bubble. The excluded area was comparable to the laser spot size used to nucleate a bubble. Only the illuminated areas within a minority carrier diffusion length of the liquid/solid interface could contribute to the gas evolution, so the presence of the bubble may lower the reaction rate. Because the bubble base blocks local sites of study from the electrolyte, this technique may not be well suited for comparing the reaction rate at various surface features, depending on the contact angle of the given semiconductor-electrolyte-gas system. Modifications to this technique could involve introducing separate nearby nucleation features such as rough Teflon fibers ${ }^{19}$ or simply scanning the sample or laser beam and measuring photocurrent at different surface features.

The variation of current seen in Fig. 3 resulting from the bubble dynamics can be understood based on two mechanisms. First, with no bubble present, the high oxygen concentration near the electrode restricted the current to be diffusion-limited, then when a bubble nucleated, it provided a large sink for the oxygen, and the reaction could proceed at a higher rate (with the caveat of the excluded electrode area). Second, the optical effects of the bubble must be considered. Because the index of refraction inside the bubble was lower than the electrolyte, the bubble acted as a diverging lens to scatter the incident laser beam. Thus, when the bubble detached and rose within the illuminating laser beam, it diverted light away from the sample, temporarily reducing the current. To illustrate the light scattering, radial intensity profiles of both a uniform and a Gaussian laser before and after scattering are shown in Fig. 6. This calculation was performed by sending many rays carrying intensities based on the value of $r$ toward a spherical bubble tangent to the electrode surface, solving Snell's law at each interface using the indexes of refraction for gas and electrolyte of 1 and 1.34, respectively, and histogramming the resultant intensities at the electrode surface. Effects of light polarization and reflection losses were not considered. In Fig. 6a, the incident laser intensity is constant as in the case of uniform illumination; it is easily seen that the bubble diverts light away from itself due to both refraction inside the bubble and total internal reflection in the liquid (and showing an apparent bright spot in the middle of the bubble). More relevant to the present experimental conditions is Fig. 6b, where an incident Gaussian laser profile with a radius smaller than the bubble radius is simply broadened by the scattering. Interestingly, the profile seen for the particular bubble radius chosen did not change as the bubble grows even larger because the increased distance from the surface offsets the lower 
bubble surface curvature. During its nucleation and growth, the bubble's lensing effects were quite evident in this experiment. In general, bubbles at photoelectrodes can hinder the flow of products and reactants, but the refraction of incident light away from the blocked area mitigates this effect.

\section{Conclusions}

By shining above-bandgap light on a semiconductor photoelectrode to form isolated, individual bubbles, we have shown that analyzing the bubble growth can provide a quantitative measure of the surface electrochemical reaction rate. Observing the evolved gas bubbles in an optical microscope can thus provide an alternative method for characterizing photoelectrode surfaces. Because $\mathrm{SrTiO}_{3}$ is quite stable against photocorrosion, the reaction rate calculated from the photocurrent using Faraday's law can be compared to the bubble growth rate, and the two methods showed quite good agreement. This method could be used for other semiconductors to characterize the relative rates of gas production and surface corrosion. However, the tendency for gas to diffuse away in solution rather than enter the bubble somewhat lowered the apparent reaction rate observed from the bubble.

The surface sites favorable for bubble nucleation are not necessarily the same surface sites with the best electron transfer rate, so we forced localized bubble nucleation by using a small laser spot with rather high irradiances. Simulating the dissolved gas profile, it was found that a supersaturation of $\sim 8$ times was necessary to nucleate a bubble on the smooth surface. However, once a bubble was nucleated, the existence of a finite contact angle meant that a significant area of the illuminated interface was excluded from the electrolyte. Thus, this method may not be well suited for comparing the reaction rates at different surface features without introducing a separate nucleation site nearby. Future work could add a nearby pit or rough hydrophobic fiber to encourage bubble nucleation near a surface feature of interest. Patterning submillimeter areas of a semiconductor photoelectrode with different surface modifications (and nearby bubble nucleation sites) would allow the use of this method to compare, on one sample, the effects of heterogeneous catalysts or morphology changes.

\section{Acknowledgments}

The Applied Materials Graduate Fellowship program and the Global Climate and Energy Project (GCEP) provided funding.

California Institute of Technology assisted in meeting the publication costs of this article.

\section{Appendix}

An analytic solution to the problem of the temperature rise in a solid when illuminated with a laser was given by Lax ${ }^{21}$ For a laser with a Gaussian intensity profile, $I=I_{0} \exp \left(-r^{2} / r_{\text {laser }}^{2}\right)$, consider the steady-state heat equation

$$
\nabla^{2} T=-\frac{G_{\text {therm }}}{K}=-\frac{I_{0}}{K} \alpha e^{-\alpha z} e^{-r^{2} / r_{\text {laser }}^{2}}
$$

where $G_{\text {therm }}$ is the energy absorbed per unit volume per second, and the sign convention for $z$ is that more positive values move away from the surface. Assuming that no heat is transferred at the surface and that all the heat is generated in an infinitesimal surface layer (essentially if the absorption depth is much smaller than the beam width), the solution obtained is

$$
\Delta T\left(r, z, r_{\text {laser }}\right)=\Delta T_{\max } N\left(r, z, r_{\text {laser }}\right)
$$

with

$$
\Delta T_{\max }=\frac{P_{\text {laser }}}{2 \sqrt{\pi} K r_{\text {laser }}}
$$

and

$$
N\left(r, z, r_{\text {laser }}\right) \rightarrow \pi_{\alpha \rightarrow \infty}^{-1 / 2} \int_{0}^{\infty} J_{0}\left(\beta r / r_{\text {laser }}\right) \exp (-\beta z) \exp \left(-\beta^{2} / 4\right) d \beta
$$

Here $P_{\text {laser }}=I_{0} \pi r_{\text {laser }}^{2}$ and $J_{0}$ is the Bessel function of the first kind of order 0 .

Using the comparison between heat flow and particle diffusion, this solution can be extended to solve the profile of dissolved gas generated in a Gaussian profile at an electrode. The steady-state diffusion equation including gas generation is

$$
\nabla^{2} c=-\frac{G_{\mathrm{gas}}}{D}
$$

where $c$ is the concentration, $G_{\text {gas }}$ is the generation rate, and $D$ is the diffusion coefficient. For the sake of comparison (recognizing that in the final solution we will take $\alpha \rightarrow \infty$ because all the gas is generated at the electrode surface), the generation of gas can be written as

$$
G_{\text {gas }}=\frac{P_{\text {laser }} \lambda \Phi}{h c \pi r_{\text {laser }}^{2} Z} \alpha e^{-\alpha z} e^{-r^{2} / r_{\text {laser }}^{2}}
$$

Here we have assumed that all the absorbed photons under a given spot generate charg carriers that then contribute to the gas generation based on the observed quantum yield. In analogy to the solution for heat flow, the dissolved product profile is then given by

$$
\begin{gathered}
c\left(r, z, r_{\text {laser }}\right)=c_{\max } N\left(r, z, r_{\text {laser }}\right) \\
C_{\max }=\frac{P_{\text {laser }} \lambda \Phi}{2 \sqrt{\pi} h c r_{\text {laser }} Z D}
\end{gathered}
$$

This solution is in the steady state, so it represents the highest concentration of dissolved gas for any time.

\section{References}

1. J. Nowotny, C. Sorrell, L. Sheppard, and T. Bak, Int. J. Hydrogen Energy, 30, 521 (2005).

2. J. Bolton, S. Strickler, and J. Connolly, Nature (London), 316, 495 (1985).

3. T. Bak, J. Nowotny, M. Rekas, and C. Sorrell, Int. J. Hydrogen Energy, 27, 991 (2002).

4. K. Kogo, H. Yoneyama, and H. Tamura, J. Phys. Chem., 84, 1705 (1980).

5. B. Kraeutler and A. Bard, J. Am. Chem. Soc., 100, 4317 (1978).

6. C. Santato, M. Ulmann, and J. Augustynski, J. Phys. Chem. B, 105, 936 (2001).

7. C. M. McShane and K.-S. Choi, J. Am. Chem. Soc., 131, 2561 (2009).

8. T. F. Jaramillo, K. P. Jorgensen, J. Bonde, J. H. Nielsen, S. Horch, and I. Chorkendorff, Science, 317, 100 (2007).

9. M. W. Kanan and D. G. Nocera, Science, 321, 1072 (2008).

10. A. J. Bard and M. V. Mirkin, Scanning Electrochemical Microscopy, Marcel Dekker, New York (2001).

11. J. Lee, H. Ye, S. Pan, and A. J. Bard, Anal. Chem., 80, 7445 (2008)

12. M. Cohen and R. Blunt, Phys. Rev., 168, 929 (1968).

13. M. Butler and D. Ginley, J. Electrochem. Soc., 125, 228 (1978).

14. A. Bocarsly, J. Bolts, P. Cummins, and M. Wrighton, Appl. Phys, Lett., 31, 568 (1977).

15. M. Wrighton, A. Ellis, P. Wolczanski, D. Morse, H. Abrahamson, and D. Ginley, $J$. Am. Chem. Soc., 98, 2774 (1976)

16. See supplementary material at http://dx.doi.org/10.1149/1.3462997 (E-JESOAN157-045009) for additional information.

17. CRC Handbook of Chemistry and Physics, D. R. Lide, Editor, CRC Press/Taylor \& Francis, Boca Raton, FL (2009).

18. M. Blander and J. Katz, AIChE J., 21, 833 (1975)

19. H. Vogt, Ö. Aras, and R. Balzer, Int. J. Heat Mass Transfer, 47, 787 (2004).

20. F. Wagner and G. Somorjai, J. Am. Chem. Soc., 102, 5494 (1980).

21. M. Lax, J. Appl. Phys., 48, 3919 (1977).

22. S. Jones, G. Evans, and K. Galvin, Adv. Colloid Interface Sci., 80, 27 (1999).

23. S. Lubetkin, Electrochim. Acta, 48, 357 (2002).

24. H. Vogt, J. Appl. Electrochem., 23, 1323 (1993).

25. N. Brandon and G. Kelsall, J. Appl. Electrochem., 15, 475 (1985).

26. H. Gerischer, in Photoelectrochemistry, Photocatalysis, and Photoreactors: Fundamentals and Developments, M. Schiavello, Editor, p. 39, Reidel, Dordrecht, The Netherlands (1985).

27. R. Darby and M. Haque, Chem. Eng. Sci., 28, 1129 (1973).

28. C. Yu, M. L. Scullin, M. Huijben, R. Ramesh, and A. Majumdar, Appl. Phys. Lett. 92, 191911 (2008)

29. H. Vogt and R. Balzer, Electrochim. Acta, 50, 2073 (2005) 